\title{
Single boson realizations of the deformed angular momentum algebra of Witten's types
}

\author{
HUANG HuaJun \& RUAN Dong* \\ Department of Physics, Tsinghua University, Beijing 100084, China
}

Received October 19, 2012; accepted December 23, 2012

By generalizing the Holstein-Primakoff realization and the Dyson realization of the Lie algebra SU(2), various realizations of the deformed angular momentum algebra $\mathcal{R}_{q, s}^{c_{0}, c_{1}, c_{2}}$, a five-parameter deformed $\mathrm{SU}(2)$ by combining Witten's two deformation schemes, are investigated in terms of the single boson and the single inversion boson respectively. For each kind, the unitary realization, the non-unitary realization, and their connection by the corresponding similarity transformation are respectively discussed.

deformed algebra, Lie algebra SU(2), boson, inversion boson

Citation: Huang H J, Ruan D. Single boson realizations of the deformed angular momentum algebra of Witten's types. Chin Sci Bull, 2013, 58: 2039-2043, doi: $10.1007 / \mathrm{s} 11434-013-5872-5$

Lie algebras have played an exciting and significant role in the development of various branches of physics and many other areas of science and technology [1-11]. In 1983, Kulish et al. [12] showed that the algebra that governs the XXZHeisenberg spin model was a deformation of the Lie algebra $\mathrm{SU}(2)$, called nowadays $\mathrm{SU}_{q}(2)$. Since then, there are many works devoted to various types of nonlinear algebras, i.e. some specific deformations of the usual Lie algebras obtained by introducing deformation parameters, due to their interesting mathematical structure and possible applications in a broad range of physical phenomena [13-27]. The nonlinear algebra to be discussed in this paper is a kind of multiparameter deformed angular momentum algebra, which generalizes Witten's first and second deformation schemes of $\mathrm{SU}(2)$ [14]. Here we denote it by $\mathcal{R}_{q, s}^{c_{0}, c_{1}, c_{2}}$, whose three elements $J_{\mu}(\mu=3,-,+)$ satisfy the following commutation relations:

$$
\begin{aligned}
& {\left[J_{3}, J_{-}\right]_{q}=-C_{0} J_{-},} \\
& {\left[J_{3}, J_{+}\right]_{\frac{1}{q}}=\frac{C_{0}}{q} J_{+},} \\
& {\left[J_{+}, J_{-}\right]_{s}=D_{0}+D_{1} J_{3}+D_{2} J_{3}^{2},}
\end{aligned}
$$

where $[X, Y]_{q} \equiv X Y-q Y X$ is a $q$-deformed commutator, $q$ and $s$ are positive real numbers, $C_{i}(i=0,1,2)$ are real numbers, and three expansion coefficients $D_{i}(i=0,1,2)$ take respectively

$$
\begin{aligned}
& D_{0}=C_{0}\left(C_{1}-C_{0} C_{2}\right), \quad D_{1}=C_{1}(1-q)+2 C_{0} C_{2} q, \\
& D_{2}=C_{2}\left(1-q^{2}\right)
\end{aligned}
$$

in order that the Casimir invariant of the type considered by Polychronakos [15] and Roček [16] exists in the limit of $s \rightarrow 1$. When $q=p^{2}, s=1, C_{0}=p, C_{1}=1 /\left(1+p^{2}\right)$, and $C_{2}=1 /\left[p\left(1+p^{2}\right)\right]$, eq. (1) reduces to Witten's first deformation, when $q=r^{2}, s=r^{4}, C_{0}=r, C_{1}=2 r^{2} /\left(1-r^{2}\right)$, and $C_{2}=0$, eq. (1) to Witten's second deformation. Furthermore, different from the quantum group $\mathrm{SU}_{q}(2)[13,19]$ and the polynomial angular momentum algebras [21-24], both the deformed commutators and the power series of $J_{3}$ appear in the algebraic structure (1). When $q=s=C_{0}=C_{1}=C_{2}=1$ (or $q=s=C_{0}=1$ and $C_{1}=C_{2}=-1$ ), $\mathcal{R}_{1,1}^{1,1,1}$ (or $\mathcal{R}_{1,1}^{1,-1,-1}$ ) becomes $\mathrm{SU}(2)$ (or its non-compact type $\mathrm{SU}(1,1)$ ). If let $q=s=C_{0}=C_{1}=1$ and $C_{2}=0$, then $\mathcal{R}_{1,1}^{1,1,0}$ becomes the standard Heisenberg-Weyl algebra [28]. Hence, $\mathcal{R}_{q, s}^{c_{0}, c_{1}, c_{2}}$ can be viewed as a type of five-parameter deformation of SU(2) (or SU $(1,1)$ ) or Heisenberg-Weyl algebra.

In this paper, we study various single boson realizations 
and single inversion boson realizations of $\mathcal{R}_{q, s}^{c_{0}, c_{1}, c_{2}}$, which are analogous to the Holstein-Primakoff realization and the Dyson realization of $\mathrm{SU}(2)$ and $\mathrm{SU}(1,1)$. This paper is arranged as follows. In Section 1, some results of boson operators and inversion boson operators are briefly reviewed. In Sections 2 and 3 , the single boson realizations and single inversion boson realizations of $\mathcal{R}_{q, s}^{c_{0}, c_{1}, c_{2}}$ are respectively studied in detail, which include unitary realizations, non-unitary realizations, and their connections. A simple discussion is given in the final section.

\section{Preliminaries}

Let $a^{+}$and $a$ ( $a$ is adjoint to $a^{+}$, i.e., $a=\left(a^{+}\right)^{\dagger}, a^{+}=(a)^{\dagger}$ ) be creation and annihilation boson operators respectively, which, together with the particle number operator $\hat{n} \equiv a^{+} a$, satisfy commutation relations [28]

$$
\left[a, a^{+}\right]=1, \quad\left[\hat{n}, a^{+}\right]=a^{+}, \quad[\hat{n}, a]=-a .
$$

The complete set of basis vectors of Fock space

$$
\mathcal{F}=\{|n\rangle \mid n=0,1,2, \ldots\}
$$

may be constructed from the vacuum state $|0\rangle$ using the definition

$$
|n\rangle=\frac{\left(a^{+}\right)^{n}}{\sqrt{n !}}|0\rangle .
$$

In fact, these vectors are normalized eigenvectors of $\hat{n}$ belonging to eigenvalue $n$, i.e.

$$
\hat{n}|n\rangle=n|n\rangle,
$$

and satisfy

$$
a^{+}|n\rangle=\sqrt{n+1}|n+1\rangle, \quad a|n\rangle=\sqrt{n}|n-1\rangle .
$$

Though the boson operators, $a$ and $a^{+}$, do not possess any inversion in a strict sense because of their singular feature, the generalized inversion of these boson operators, denoted by $a^{-1}$ and $\left(a^{+}\right)^{-1}$ respectively, may be defined by their action on the basis vectors of Fock space [29-31]:

$$
\begin{aligned}
a^{-1}|n\rangle & =\frac{1}{\sqrt{n+1}}|n+1\rangle, \\
\left(a^{+}\right)^{-1}|n\rangle & =\left(1-\delta_{n, 0}\right) \frac{1}{\sqrt{n}}|n-1\rangle .
\end{aligned}
$$

In fact, $a^{-1}$ is only the right inversion of $a$ since it satisfies

$$
a a^{-1}=I \text {. }
$$

However, $a^{-1} a$ is not unity $(I)$ but is given by

$$
a^{-1} a=I-|0\rangle\langle 0|,
$$

where $|0\rangle\langle 0|$ is the projection operator on vacuum. Similarly, $\left(a^{+}\right)^{-1}$ is only the left inversion of $a^{+}$. As is seen, $a^{-1}$ behaves as the creation operator, while $\left(a^{+}\right)^{-1}$ as the annihilation operator.

By direct calculations, it is easy to obtain the following commutation relations:

$$
\begin{aligned}
{\left[\left(a^{+}\right)^{-1}, a^{+}\right] } & =|0\rangle\langle 0|, \\
{\left[\hat{n},\left(a^{+}\right)^{-1}\right] } & =-\left(a^{+}\right)^{-1}, \\
{\left[\hat{n}, a^{-1}\right] } & =a^{-1} .
\end{aligned}
$$

\section{Boson realizations of $\mathcal{R}_{q, s}^{c_{0}, c_{1}, c_{2}}$}

The single boson realizations of $\mathcal{R}_{q, s}^{c_{0}, c_{1}, c_{2}}$ may be chosen in the following form:

$$
\begin{aligned}
& B^{(k)}\left(J_{3}\right)=h^{(k)}(\hat{n}), \\
& B^{(k)}\left(J_{+}\right)=f^{(k)}(\hat{n}) a^{k}, \\
& B^{(k)}\left(J_{-}\right)=\left(a^{+}\right)^{k} g^{(k)}(\hat{n}),
\end{aligned}
$$

where $k$ is an integer, $h^{(k)}(\hat{n}), f^{(k)}(\hat{n})$, and $g^{(k)}(\hat{n})$, being real operator functions of $\hat{n}$ only, need determining by the commutation relations (1). The action of $B^{(k)}\left(J_{ \pm}\right)$on the basis vector $|n\rangle$ of the Fock space $\mathcal{F}$ leads to $|n \mp k\rangle \sim B^{(k)}\left(J_{ \pm}\right)|n\rangle$.

The boson realizations $B^{(k)}\left(J_{\mu}\right)(\mu=3, \pm)$ are unitary if they satisfy

$$
\begin{aligned}
& {\left[B^{(k)}\left(J_{3}\right)\right]^{\dagger}=B^{(k)}\left(J_{3}\right),} \\
& {\left[B^{(k)}\left(J_{ \pm}\right)\right]^{\dagger}=B^{(k)}\left(J_{\mp}\right) .}
\end{aligned}
$$

We call eq. (10) the unitary conditions of the boson realization.

For the case of $k=1$. Using the first or second equation of eq. (1), we may obtain the single-variable difference equation satisfied by $h^{(1)}(\hat{n})$

$$
h^{(1)}(\hat{n})-\frac{1}{q} h^{(1)}(\hat{n}+1)=\frac{C_{0}}{q},
$$

with the help of the relations

$$
\begin{aligned}
\left(a^{+}\right)^{k} f(\hat{n}) & =f(\hat{n}-k)\left(a^{+}\right)^{k}, \\
a^{k} f(\hat{n}) & =f(\hat{n}+k) a^{k} .
\end{aligned}
$$

The solution of eq. (11) reads

$$
h^{(1)}(\hat{n})=\frac{C_{0}}{q-1}\left(1-q^{\hat{n}-\alpha}\right),
$$

where $\alpha$ is an arbitrary real number. It is worth mentioning that $h^{(1)}(\hat{n})$ is independent of the parameters $s, C_{1}$, and $C_{2}$.

The third equation of eq. (1) requires that $f^{(1)}(\hat{n})$ and $g^{(1)}(\hat{n})$ satisfy the following difference equation:

$$
\begin{aligned}
& (\hat{n}+1) f^{(1)}(\hat{n}) g^{(1)}(\hat{n})-s \hat{n} f^{(1)}(\hat{n}-1) g^{(1)}(\hat{n}-1) \\
& =C_{0}\left(C_{1}-C_{0} C_{2}\right)+\left[C_{1}(1-q)+2 C_{0} C_{2} q\right] h^{(1)}(\hat{n}) \\
& \quad+C_{2}\left(1-q^{2}\right)\left[h^{(1)}(\hat{n})\right]^{2}
\end{aligned}
$$


Note that the functions $f^{(1)}(\hat{n})$ and $g^{(1)}(\hat{n})$ do not appear separately but only appear as their product $f^{(1)}(\hat{n}) g^{(1)}(\hat{n})$.

Inserting eq. (13) into eq. (14) and solving it, we obtain

$$
\begin{aligned}
f^{(1)}(\hat{n}) g^{(1)}(\hat{n})= & \frac{C_{0} q^{-2 \alpha}}{\hat{n}+1}\left[\left(C_{1}+\frac{2 C_{0} C_{2}}{q-1}\right) q^{\alpha} D(1, \hat{n}+1)\right. \\
& \left.-\frac{C_{0} C_{2}(q+1)}{q-1} D(2, \hat{n}+1)\right]
\end{aligned}
$$

where $D(x, y)$ is defined as

$$
D(x, y)=\frac{q^{x y}-s^{y}}{q^{x}-s} .
$$

This solution shows that we may have some freedom in the choice of the functions $f^{(1)}(\hat{n})$ and $g^{(1)}(\hat{n})$.

(i) The unitary boson realization. The second equation of eq. (10) requires that $f^{(1)}(\hat{n})=g^{(1)}(\hat{n})$, then solving eq. (15), we may obtain from eq. (9) a kind of unitary single boson realization

$$
\begin{aligned}
& \breve{B}^{(1)}\left(J_{3}\right)=\frac{C_{0}}{q-1}\left(1-q^{\hat{n}-\alpha}\right), \\
& \breve{B}^{(1)}\left(J_{+}\right)= \\
& \sqrt{\frac{q^{-2 \alpha} C_{0}}{\hat{n}+1}\left[\left(C_{1}+\frac{2 C_{0} C_{2}}{q-1}\right) q^{\alpha} D(1, \hat{n}+1)-\frac{C_{0} C_{2}(q+1)}{q-1} D(2, \hat{n}+1)\right]} a, \\
& \breve{B}^{(1)}\left(J_{-}\right)= \\
& a^{+} \sqrt{\frac{q^{-2 \alpha} C_{0}}{\hat{n}+1}\left[\left(C_{1}+\frac{2 C_{0} C_{2}}{q-1}\right) q^{\alpha} D(1, \hat{n}+1)-\frac{C_{0} C_{2}(q+1)}{q-1} D(2, \hat{n}+1)\right]} .
\end{aligned}
$$

In order to obtain the real boson realization, the values of $n$ in the matrix elements of $\breve{B}^{(1)}\left(J_{ \pm}\right)$in the Fock space $\mathcal{F}=$ $\{|n\rangle \mid n=0,1,2, \ldots\}$ need limiting for the given $\left\{q, s, C_{0}, C_{1}\right.$, $\left.C_{2}\right\}$. When $q=s=C_{0}=C_{1}=C_{2}=1$, eq. (17) becomes the Holstein-Primakoff realization of SU(2) [32]. Hence, we call $\breve{B}^{(1)}\left(J_{\mu}\right)$ the Holstein-Primakoff-like realization of $\mathcal{R}_{q, s}^{c_{0}, c_{1}, c_{2}}$.

(ii) The non-unitary boson realization. If the unitary conditions (10) need not satisfying, it follows from eq. (15) that the convenient choice, for example, $g(\hat{n})=1$, may immediately give rise to a kind of non-unitary single boson realization:

$$
\begin{aligned}
\bar{B}^{(1)}\left(J_{3}\right)= & \frac{C_{0}}{q-1}\left(1-q^{\hat{n}-\alpha}\right), \\
\bar{B}^{(1)}\left(J_{+}\right)= & \frac{C_{0} q^{-2 \alpha}}{\hat{n}+1}\left[\left(C_{1}+\frac{2 C_{0} C_{2}}{q-1}\right) q^{\alpha} D(1, \hat{n}+1)\right. \\
& \left.-\frac{C_{0} C_{2}(q+1)}{q-1} D(2, \hat{n}+1)\right] a, \\
\bar{B}^{(1)}\left(J_{-}\right)= & a^{+} .
\end{aligned}
$$

When $q=s=C_{0}=C_{1}=C_{2}=1$, eq. (18) becomes the standard Dyson realization of SU(2) [33]. Hence, we call $\bar{B}^{(1)}\left(J_{\mu}\right)$ the Dyson-like realization of $\mathcal{R}_{q, s}^{c_{0}, c_{1}, c_{2}}$. Different from the Holstein-Primakoff-like realization (17), no square-root symbol appears in eq. (18) so that the Dyson-like realization may not only avoid the convergence questions associated with the expansion of square-root symbol but also make the values of $n$ in $\mathcal{F}=\{|n\rangle \mid n=0,1,2, \cdots\}$ unlimited.

(iii) Unitarization of the non-unitary realization. It is not difficult to find that the non-unitary realization $\bar{B}^{(k)}\left(J_{\mu}\right)$ may be related to the unitary realization $\breve{B}^{(k)}\left(J_{\mu}\right)$ by a similarity transformation $S$, i.e.

$$
S \bar{B}^{(k)}\left(J_{\mu}\right) S^{-1}=\breve{B}^{(k)}\left(J_{\mu}\right) .
$$

In general, $S$ is an operator function with respect to $\left\{a, a^{+}, \hat{n}\right\}$. Using the unitary condition $\breve{B}^{(k)}\left(J_{+}\right)=\left(\breve{B}^{(k)}\left(J_{-}\right)\right)^{\dagger}$, we obtain from eq. (19)

$$
U^{-1}\left(\bar{B}^{(k)}\left(J_{-}\right)\right)^{\dagger} U=\bar{B}^{(k)}\left(J_{+}\right),
$$

where $U \equiv S^{\dagger} S$. Note that $\bar{B}^{(k)}\left(J_{3}\right)$ is already Hermitian, so we call eq. (20) the unitarization equation of the Dyson-like realization.

Now let us calculate the explicit expression of $S_{1}$, which transforms the Dyson-like realization $\vec{B}^{(1)}\left(J_{\mu}\right)$ to the HolsteinPrimakoff-like realization $\breve{B}^{(1)}\left(J_{\mu}\right)$. Owing to the fact that $\bar{B}^{(k)}\left(J_{3}\right)=\breve{B}^{(k)}\left(J_{3}\right)$ for the given integer $k$, eq. (19) implies that $S_{1}$ commutes with $J_{3}$ and is at most the function of $\hat{n}$, i.e. $S_{1}(\hat{n})$. Thus, calculating the matrix element of eq. (20) between the basis vectors $\langle n-1|$ and $|n\rangle$ and using eq. (18), we may derive the equation satisfied by $\left\langle n\left|S_{1}(\hat{n})\right| n\right\rangle \equiv S_{1}(n)$. i.e.

$$
\begin{gathered}
n\left\langle n\left|S_{1}(\hat{n})\right| n\right\rangle^{2}=C_{0} q^{-2 \alpha}\left[\left(C_{1}+\frac{2 C_{0} C_{2}}{q-1}\right) q^{\alpha} D(1, \hat{n})\right. \\
\left.-\frac{C_{0} C_{2}(q+1)}{q-1} D(2, \hat{n})\right]\left\langle n-1\left|S_{1}(\hat{n})\right| n-1\right\rangle^{2} .
\end{gathered}
$$

Solving eq. (21) with the initial condition $\left\langle 0\left|S_{1}(\hat{n})\right| 0\right\rangle=\kappa_{0}$ $\left(\kappa_{0}\right.$ is a real number) gives

$$
\begin{aligned}
S_{1}(n)= & \kappa_{0}\left[\frac{-C_{0}}{(q-1)(q-s)\left(q^{2}-s\right)}\right]^{n / 2} \\
& \cdot \prod_{l=0}^{n-1} \frac{1}{\sqrt{l+1}}\left\{C_{1} q^{-\alpha}(q-1)\left(q^{2}-s\right)\left(q^{l+1}-s^{l+1}\right)\right. \\
& +C_{0} C_{2} q^{-\alpha}(q-s)\left[2(q+s)\left(q^{l+1}-s^{l+1}\right)\right. \\
& \left.\left.-q^{-\alpha}(q+1)\left(q^{2(l+1)}-s^{l+1}\right)\right]\right\}^{1 / 2} .
\end{aligned}
$$

Using eq. (5), $S_{1}(n)$ may be written as the operator function $S_{1}(\hat{n})$ by replacing directly $n \rightarrow \hat{n}$ in eq. (22).

\section{Inversion boson realizations of $\mathcal{R}_{q, s}^{c_{0}, c_{1}, c_{2}}$}

For the case of $k=-1$. Substitution of eq. (9) in eq. (1) yields the following two equations satisfied by $h^{(-1)}(\hat{n})$ and $f^{(-1)}(\hat{n}) g^{(-1)}(\hat{n})$, respectively:

$$
h^{(-1)}(\hat{n})-\frac{1}{q} h^{(-1)}(\hat{n}-1)=\frac{C_{0}}{q},
$$

and

$$
\frac{1}{\hat{n}} f^{(-1)}(\hat{n}) g^{(-1)}(\hat{n})-\frac{s}{\hat{n}+1} f^{(-1)}(\hat{n}+1) g^{(-1)}(\hat{n}+1)
$$




$$
\begin{aligned}
= & C_{0}\left(C_{1}-C_{0} C_{2}\right)+\left[C_{1}(1-q)+2 C_{0} C_{2} q\right] h^{(-1)}(\hat{n}) \\
& +C_{2}\left(1-q^{2}\right)\left[h^{(-1)}(\hat{n})\right]^{2} .
\end{aligned}
$$

Their solutions are respectively

$$
\begin{aligned}
h^{(-1)}(\hat{n})= & \frac{C_{0}}{q-1}\left(1-q^{-\hat{n}-1}\right), \\
f^{(-1)}(\hat{n}) g^{(-1)}(\hat{n})= & \hat{n} C_{0}\left[\left(C_{1}+\frac{2 C_{0} C_{2}}{q-1}\right) D(1,-\hat{n})\right. \\
& \left.-\frac{C_{0} C_{2}(q+1)}{q-1} D(2,-\hat{n})\right],
\end{aligned}
$$

where $D(x, y)$ is given by eq. (16).

It follows from eq. (26) that we obtain the explicit expressions for the unitary single inversion boson realization by taking $f^{(-1)}(\hat{n})=g^{(-1)}(\hat{n})$, i.e.

$$
\begin{aligned}
& \breve{B}^{(-1)}\left(J_{3}\right)=\frac{C_{0}}{q-1}\left(1-q^{-\hat{n}-1}\right), \\
& \breve{B}^{(-1)}\left(J_{+}\right)= \\
& \sqrt{\hat{n} C_{0}\left[\left(C_{1}+\frac{2 C_{0} C_{2}}{q-1}\right) D(1,-\hat{n})-\frac{C_{0} C_{2}(q+1)}{q-1} D(2,-\hat{n})\right]} a^{-1}, \\
& \breve{B}^{(-1)}\left(J_{-}\right)= \\
& \left(a^{+}\right)^{-1} \sqrt{\hat{n} C_{0}\left[\left(C_{1}+\frac{2 C_{0} C_{2}}{q-1}\right) D(1,-\hat{n})-\frac{C_{0} C_{2}(q+1)}{q-1} D(2,-\hat{n})\right]},
\end{aligned}
$$

and the non-unitary one by taking $g^{(-1)}(\hat{n})=1$, i.e.

$$
\begin{aligned}
\bar{B}^{(-1)}\left(J_{3}\right)= & \frac{C_{0}}{q-1}\left(1-q^{-\hat{n}-1}\right), \\
\bar{B}^{(-1)}\left(J_{+}\right)= & \hat{n} C_{0}\left[\left(C_{1}+\frac{2 C_{0} C_{2}}{q-1}\right) D(1,-\hat{n})\right. \\
& \left.\quad-\frac{C_{0} C_{2}(q+1)}{q-1} D(2,-\hat{n})\right] a^{-1}, \\
\bar{B}^{(-1)}\left(J_{-}\right)= & \left(a^{+}\right)^{-1} .
\end{aligned}
$$

The above two kinds of inversion boson realizations are unfamiliar to us. Now let us see the corresponding results for a simple case $\mathcal{R}_{1,1}^{c_{0}, c_{1}, c_{2}}$.

Taking $q=s=1$ in eq. (1), introducing the parameter $\lambda=C_{1} / C_{2}$, and rescaling $J_{ \pm}$, the commutation relations of $\mathcal{R}_{1,1}^{c_{0}, c_{1}, c_{2}}$ read

$$
\begin{aligned}
{\left[J_{3}, J_{-}\right] } & =-C_{0} J_{-}, \\
{\left[J_{3}, J_{+}\right] } & =C_{0} J_{+}, \\
{\left[J_{+}, J_{-}\right] } & =(\lambda-1)+2 J_{3} .
\end{aligned}
$$

In fact $\mathcal{R}_{1,1}^{c_{0}, c_{1}, c_{2}}$ is a two-parameter deformed algebra. Thus, from eq. (27) we may immediately obtain the single inversion boson realization of $\mathcal{R}_{1,1}^{c_{0}, c_{1}, c_{2}}$ :

$$
\begin{aligned}
& \breve{B}_{\mathcal{R}_{1,1}}\left(J_{3}\right)=C_{0}(\hat{n}+1) \\
& \breve{B}_{\mathcal{R}_{1,1}}\left(J_{+}\right)=\hat{n} \sqrt{-C_{0}\left(\lambda+C_{0} \hat{n}\right)} a^{-1} \\
& \breve{B}_{\mathcal{R}_{1,1}}\left(J_{-}\right)=\left(a^{+}\right)^{-1} \hat{n} \sqrt{-C_{0}\left(\lambda+C_{0} \hat{n}\right)}
\end{aligned}
$$

For the realization (30), the acting space of $\breve{B}_{\mathcal{R}_{1,1}}\left(J_{\mu}\right)(\mu=$ $3,-,+)$ may be certain subspace of the Fock space $\mathcal{F}$, in which $n$ needs limiting in order that the values of the square roots appeared in the matrix elements $\left\langle n \pm 1\left|\breve{B}_{\mathcal{R}_{1,1}}\left(J_{ \pm}\right)\right| n\right\rangle$ must be greater than or equal to zero, i.e. $n$ has to satisfy the constraint equation $-C_{0}\left(\lambda+C_{0} n\right) \geqslant 0$. If $C_{0}<0$, then $n$ may take $0,1,2, \ldots,\left\lceil\lambda /\left|C_{0}\right|\right\rfloor$ (where $\lceil x\rfloor$ means integer part of $x$ ). If $C_{0}>0$, then $n$ may take $0,1,2, \ldots,\left\lceil-\lambda / C_{0}\right\rfloor$. Their corresponding acting spaces are finite-dimensional. Especially, when $C_{0}=\lambda=1$, eq. (29) becomes the commutation relations of SU(2), then the square roots in the matrix elements $\left\langle n \pm 1\left|\breve{B}_{\mathrm{Su} 2}\left(J_{ \pm}\right)\right| n\right\rangle$ are pure imaginary or null.

Unitarization of the non-unitary realization. Calculating the matrix element of eq. (20) between the basis vectors $\langle n+1|$ and $|n\rangle$ and using eq. (28), we obtain the equation satisfied by $\left\langle n\left|S_{2}(\hat{n})\right| n\right\rangle \equiv S_{2}(n)$ of the similarity transformation $S_{2}(\hat{n})$, which transforms the non-unitary realization $\bar{B}^{(-1)}\left(J_{\mu}\right)$ to the unitary realization $\breve{B}^{(-1)}\left(J_{\mu}\right)$ :

$$
\begin{gathered}
\left\langle n\left|S_{2}(\hat{n})\right| n\right\rangle^{2}=(n+1) C_{0}\left[\left(C_{1}+\frac{2 C_{0} C_{2}}{q-1}\right) D(1,-n-1)\right. \\
\left.-\frac{C_{0} C_{2}(q+1)}{q-1} D(2,-n-1)\right]\left\langle n+1\left|S_{2}(\hat{n})\right| n+1\right\rangle^{2} .
\end{gathered}
$$

Its solution is

$$
\begin{aligned}
S_{2}(n)= & \varepsilon_{0}\left[\frac{-C_{0}}{(q-1)(q-s)\left(q^{2}-s\right)}\right]^{-n / 2} \\
& \cdot \prod_{l=0}^{n-1} \frac{q^{l+1} s^{(l+1) / 2}}{\sqrt{l+1}}\left\{-C_{1}(q-1)\left(q^{2}-s\right) q^{l+1}\left(q^{l+1}-s^{l+1}\right)\right. \\
& +C_{0} C_{2}\left[(q-1)(q+s) q^{2(l+1)}+(q+1)(q-s) s^{l+1}\right. \\
& \left.\left.-2\left(q^{2}-s\right) q^{l+1} s^{l+1}\right]\right\}^{-1 / 2},
\end{aligned}
$$

with the initial condition being $\left\langle 0\left|S_{2}(\hat{n})\right| 0\right\rangle=\varepsilon_{0}\left(\varepsilon_{0}\right.$ is a real number).

\section{Conclusion}

In this paper we have obtained the single boson and the single inversion boson realizations of the five-parameter deformed algebra $\mathcal{R}_{q, s}^{c_{0}, c_{1}, c_{2}}$ by generalizing the Holstein-Primakoff realization and the Dyson realization of SU(2). Hence, using these results, the representations of $\mathcal{R}_{q, s}^{c_{0}, c_{1}, c_{2}}$ in the Fock space $\mathcal{F}$ may be directly obtained using eqs. (5)-(7), which are not given here. Comparing the boson realization for $\alpha=0$ (see eqs. (13) and (15)) with the inversion boson realization (see eqs. (25) and (26)), there are simple replacements: $\hat{n}+1 \rightarrow-\hat{n}$ in the functions $h(\hat{n})$ and $D(x, \hat{n})$, and $\hat{n}+1 \rightarrow 1 / \hat{n}$ elsewhere. We have revealed the fact that the non-unitary realizations and the unitary ones may be related by the similarity transformations, which have been obtained by solving the corresponding unitarization equations. 
This work was supported by the National Basic Research Program of China (2009CB929402).

1 Georgi H. Lie Algebras in Particle Physics: From Isospin to Unified Theories. Boulder: Westview Press, 1999

2 Sun H Z, Han Q Z. Lie Algebras, Lie Superalgebras, and Their Applications in Physics (in Chinese). Beijing: Peking University Press, 1999

3 Chen X J, Zhang M, Sun H Z, et al. The interacting boson model II and its U(5) limit. Sci Sin Ser A, 1982, 25: 834-844

4 Zhang M, Chen X J, Sun H Z, et al. The O(6) dynamical symmetry in even-even nuclei. Sci Sin Ser A, 1982, 25: 952-959

5 Sun H Z, Han Q Z, Chen X J, et al. Rotation limit of the neutron proton interacting boson model II. Sci Sin Ser A, 1982, 25: 1165-1171

$6 \mathrm{Wu} \mathrm{G} \mathrm{Z}$. An algebraic approach to the internal rotation of molecules. Chin Sci Bull, 1986, 31: 1323-1323

7 Feng D T, Ding S L, Wang M S. Dynamical Lie algebra method for highly excited vibrational state of asymmetric linear tetratomic molecules. Sci China Ser G-Phys Mech Astron, 2003, 46: 602-609

8 Guo H Y, Huang C G, Wu H T, et al. The principle of relativity, kinematics and algebraic relations. Sci China Phys Mech Astron, 2010, 53: 591-597

9 Tian C. Lie algebra of the symmetries of Burgers equation. Chin Sci Bull, 1987, 32: 1576-1576

$10 \mathrm{Xu}$ J B, Gao J, Gao X C. Time evolution and Aharonov-Anandan phase for system with dynamical semisimple Lie algebra. Chin Sci Bull, 1992, 37: 1958-1958

11 Yuan L W, Lu G N, Luo W, et al. Geometric algebra method for multidimensionally-unified GIS computation. Chin Sci Bull, 2012, 57: 802-811

12 Kulish P P, Reshetikhin N Y. Quantum linear problem for the sineGordon equation and higher representations. J Sov Math, 1983, 23: 2435-2441

13 Majid S. Foundations of Quantum Group Theory. Cambridge: Cambridge University Press, 1995

14 Witten E. Gauge theories, vertex models, and quantum groups. Nucl Phys B, 1990, 330: 285-346

15 Polychronakos A P. A classical realization of quantum algebras. Mod Phys Lett A, 1990, 5: 2325-2334
16 Roček M. Representation theory of the nonlinear SU(2) algebra. Phys Lett B, 1991, 255: 554-557

17 Ge M L, Liu G Q, Zhao H K. Braid group and quantum group of Z(N) model. Chin Sci Bull, 1993, 38: 684

18 Sun C P, Li W. Generalized quasi-exactly-solvable quantal problems, the representations and differential realization of general deformation of su(2). Commun Theor Phys, 1993, 19: 191-198

19 Biedenharn L C, Lohe M A. Quantum Group Symmetry and q-Tensor Algebras. Singapore: World Scientific, 1995

20 Bonatsos D, Daskaloyannis C. Quantum groups and their applications in nuclear physics. Prog Part Nucl Phys, 1999, 43: 537-618

21 Beckers J, Brihaye Y, Debergh N. On realizations of nonlinear Lie algebras by differential operators. J Phys A, 1999, 32: 2791-2803

22 Ruan D, Wang F, Tu C C, et al. Indecomposable representations of the nonlinear angular momentum algebra of quadratic type. Commun Theor Phys, 2000, 34: 643-648

23 Ruan D, Jia Y F, Ruan W. Boson and differential realizations of polynomial angular momentum algebra. J Math Phys, 2001, 42: 2718-2724

24 Ruan D, Wu C, Sun H Z. Single boson realizations of the Higgs algebra. Commun Theor Phys, 2003, 40: 73-76

25 Curado E M F, Rego-Monteiro M A, Nazareno H N. Heisenberg-type structures of one-dimensional quantum Hamiltonians. Phys Rev A, 2001, 64: 012105

26 Ferrara S, Ivanov E, Lechtenfeld O, et al. Non-anticommutative chiral singlet deformation of $\mathrm{N}=(1,1)$ gauge theory. Nucl Phys B, 2005, 704: 154-180

27 Su G Z, Cai S K, Chen J C. Bose-Einstein condensation of a relativistic q-deformed Bose gas. J Phys A, 2008, 41: 045007

28 Biedenharn L C, Louck J D. Angular Momentum in Quantum Physics. Massachusetts: Addison-Wesley Press, 1981

29 Dirac P A M. Lectures on Quantum Field Theory. New York: Academic Press, 1966

30 Mehta C L, Roy A K, Saxena G M. Eigenstates of two-photon annihilation operators. Phys Rev A, 1992, 46: 1565-1572

31 Fan H Y. Inverse operators in Fock space studied via a coherent-state approach. Phys Rev A, 1993, 47: 4521-4523

32 Holstein T, Primakoff H. Field dependence of the intrinsic domain magnetization of a ferromagnet. Phys Rev, 1940, 58: 1098-1113

33 Dyson J F. General theory of spin-wave interactions. Phys Rev, 1956, 102: $1217-1230$

Open Access This article is distributed under the terms of the Creative Commons Attribution License which permits any use, distribution, and reproduction in any medium, provided the original author(s) and source are credited. 